\title{
Educational Technologies to Support New Directions in Teaching Practice
}

\author{
Ross Yates, Senior Member, IACSIT
}

\begin{abstract}
The advancement in ubiquitous computing has made the process of learning more accessible to many students, particularly those in geographically dispersed locations around the world. The recent introduction of Massive Open Online Courses (MOOCs) and other disruptive technologies into the eLearning landscape has also brought into question formerly held pedagogical notions and in many instances, heralded changes aligned with both student expectations and technological drivers. This transition has challenged not only teaching practices, but also the vehicle with which to deliver the learning content. The Learning Management System (LMS) has hitherto been a popular tool of use to deliver a range of, mostly static, learning artefacts in educational institutions, often as an "online" and "supplementary" medium to support face-to-face offerings. The LMS is now expected to align with emerging technological developments such as mobile computing and synchronous lecture engagement activities within the classroom environment. This paper explores the recent advancement of teaching practice and proposes methods by which the LMS can evolve to meet the needs and expectations of its users.
\end{abstract}

Index Terms-Learning management systems, disruptive technology, evolution, mobile learning.

\section{INTRODUCTION}

The ability of custodians of educational technologies to identify threats and opportunities and respond to them through the provision of enhanced services is becoming increasingly important within the contemporary learning landscape. The response to the need to change will need to be carried out in concert with the collaborative efforts of all stakeholders if it is to be successful. With the increasing number of new educational systems becoming available a heightened emphasis is placed on the core functionality of the LMS, in particular, to respond to new directions in educational practice or risk being replaced by another, more usable electronic system.

In providing context for the digital environment at this time, it is important to note that in 2004 eLearning was in its infancy and the associated technical infrastructure was not able to support bandwidth-intensive components such as rich media, thus making for a bland learning experience. User resistance to the use of an LMS characterised this learning landscape, both in relation to process change from paper-based practices to an online, digital medium, as well as the use of computer systems which required new skills to operate.

In an early definition of the characteristics of eLearning (as

Manuscript received April 25, 2013; revised June 30, 2013.

Ross Yates is with the University of Western Australia, Australia (e-mail: ross.yates@uwa.edu.au). supported by an LMS) and as distinct from classroom-based learning Zhang, Zhao, Zhou and Jay F. Nunamaker [1] suggested that a significant disadvantage of eLearning advantages existed in its "lack of immediate asynchronous feedback". Noting other disadvantages of eLearning, [1] proposes that the experience of eLearning was, in itself, "not comfortable to some people" and may lead to "frustration, anxiety and confusion". These early concerns may have been attributable to the general resistance on the part of people to move into the digital arena from a formerly paper-based environment, and to some extent, a lack of understanding for emerging technologies which often required changes to existing business processes.

Since the early incorporation of the LMS in the mainstream educational curriculum over a decade ago, their evolution has largely been slow. This has been attributable, at least in part, to elements such as bandwidth constraints and computer processing limitations as well as the overall expense of owning a personal computer. As computers became more affordable and bandwidth capability increased over networks, so too did accessibility to learning content such as video-based material and many other asynchronous services. The rapid expansion of large-scale classes, together with the introduction of new concepts such as "flipped classrooms" where classroom contact is minimised and online teaching and research provide context and preparation for the lecture, have focussed the learning spotlight on educational technologies as vehicles for content delivery.

Moving to the contemporary LMS environment, characterised by significant advances in pedagogical practice, for example, increased emphasis on the use of electronic systems and online collaboration, combined with a greater depth of understanding of student requirements, notable changes have occurred for both academic staff and students alike. Whilst advances have been made in some areas of learning, this is not always the case. In many instances, lecturers erroneously presuppose that students are self-motivated and thus readily participate and engage in classroom activities [2]. Whilst this assertion may have some validity, the underpinning requirement should be for lecturers to provide a more learning-centric environment in which students can learn. Such a step could include the "rethinking" of the notion of learning in general and, to some extent, this is evident with the advancement of new "disruptive" technologies like MOOCs, flipped classrooms and social learning which shift the driver of learning to that of online practice.

Expanding on the notion of the dynamic nature of the contemporary learning space, Ding [3] suggests that: "Technologies do not provide magical solutions to eLearning encounters; it depends on how people and organisations 
make use of them effectively to add value to learning communities". This assertion introduces the need for an interactional element that extends beyond the early asynchronous design of learning management systems. Such a large-scale shift in key functionality of a traditional LMS brings into question whether or not it is capable of evolving to the extent that drivers such as student expectations and willingness to engage in learning practices will be met.

\section{DistAnCE LEARNING AS ANTECEDENT TO THE LEARNING MANAGEMENT SYSTEM}

The concept of learning taking place outside the traditional "classroom" environment is not new and distance education has witnessed several conceptual milestones prior to evolving into the current technology-driven model that is in widespread use, particularly in educational institutions. One of the earliest distance education concepts was "correspondence teaching" and one of the first examples was implemented by Isaac Pitman in England in 1840 Mahnegar [4]. Mahnegar cites later iterations of the original model which introduced elements such as multimedia and encompassed audio and video tapes around 1969. Then, later in the 1980s, the arrival of personal computers changed the learning-space landscape still further, paving the way for the introduction of modern educational technologies and internet-based learning systems.

Tim Berners-Lee, who invented the World Wide Web (WWW) in the 1990s, provided a convenient mechanism by which collaboration between people (most notably students) could occur [5]. The early learning functionality widely offered by WWW was described by Downes [6] as being primarily a "...system that organizes and delivers online courses - the learning management system (LMS)". Elaborating on the concept of an LMS, Downes highlighted the following characteristics inherent in their design: "Content is ... delivered either completely online or in conjunction with more traditional seminars, to cohorts of students, led by an instructor, following a specified curriculum to be completed at a predetermined pace".

Looking ahead, Downes predicted changes in key areas of the original model of an LMS which included "....instant responses and feedback", "on-demand access to online material" as well as "to be in constant contact with friends" [7]. Downes further noted a change in the nature of the WWW, from a largely static Web 1.0 framework, to a more direct engaging, interactive and collaborative structure termed Web 2.0 [7]. This shift in functionality was described as moving from "The Read Web", to the "Read-Write Web" and aligned with Berners-Lee's earlier vision of a more "linked" Web [8].

The Web 2.0 environment witnessed the birth of social networking, blogs and podcasting with sites such as Orkut and LinkedIn emerging to address consumer demand. Downes [7] suggested that these capabilities should be viewed more as "... an attitude not a technology" placing a cultural emphasis on the manner in which people were now viewing and interacting with others online. The second generation Web was a likely driver for advancements in LMSs as a similar second generation E-Learning 2.0, characterised by a "shared domain of interest" as an element of a "community of practice" and where members "develop a shared repertoire of resources" and employing similar collaborative tools available within Web 2.0 [7].

The realisation of Downes' predictions within the boundaries of common online practice has highlighted a clear, evolutionary path on which future changes may also act as drivers to shape future iterations of educational systems and, in particular, the role of the LMS. The trend for the interaction and collaboration among users is likely to continue with more synchronous activities and closer engagement between users being key characteristics of that environment.

\section{DRIVERS OF CHANGE}

Two key findings identified in a 2010 think tank on the future of the LMS were that the current LMS design has failed to adapt to changing demands for learning, and that students require a more personalised learning experience [9]. Taylor [10] has posed the question: "Can the LMS survive in a world where ... much learning comes via our interactions with others?" These related elements highlight issues with the current state of the LMS and the challenge is made to develop solutions to ensure its ongoing sustainability. Failure to do so would result in it being replaced by one or other more applicable educational technologies. Against a backdrop of significant changes in the educational sector brought about by new thinking such as the "flipped classrooms", the role of the LMS is further heightened, and highlighted as an integral tool in contemporary teaching.

A recent study has demonstrated that as numbers of student enrolments at universities increase, a greater reliance is placed on e-Learning systems to support that demand [11]. The exponential growth in student numbers may not always be met with additional lecturers to maintain initial student to lecturer ratios (particularly when cost savings measures are in place). This situation may act as a trigger for universities to seek more innovative and less labour-intensive alternatives to support the teaching and learning process. Many prominent educational institutions have begun to explore so called "disruptive technologies", some of which offer a significant departure from the traditional classroom-based pedagogies with peer support and evaluations, webinars and self-directed learning as key elements.

The year 2012 has been described by Pappano [12] as the "Year of the MOOC" (Massive Open Online Courses). During this time a number of well-known universities such as Harvard and Stanford provided free online unit offerings to students. With Stanford having received 160,000 student enrolments in one unit [13], the dynamics of the lecture format, assessment structure and content delivery required a new approach to manage the increased numbers as it was clearly incompatible with previous, traditional classroom-based teaching practices.

It is no surprise, therefore, that the rapid growth of the MOOC industry has created a significant urgency to review the current teaching framework in many educational institutions and also to align teaching methods more closely with the changing expectations of the students with whom 
they engage. Gartner notably provides future predictions for 2013 on the possible influences of MOOCs in the university sector and posits that "with its massive scale with regard to students, MOOCs can become an adaptive learning war zone for learning data" [14]. Gartner further predicts that the effect of the "disruption" occurring in the education sector brought about by recent changes is "...expanding the education ecosystem beyond traditional lecture halls and classrooms to accommodate learners' preferences for time, place, style and previous levels of attainment".

In addition, Gartner suggests the new learning landscape will be characterised by big data repositories, formative assessments and driven by "... analysis solutions that take the student out of the context on the campus, region or even continent to develop a more individual view of the learner" [14]. This understanding, Gartner suggests, will be acquired through knowledge gleaned from personal profiles based on the characteristics of the user's information in the context of what is known about others with the same personal attributes, environment and experience. In support of that prediction, it further suggests that "institutions like Harvard University have already stated that they employ MOOCs partly because of the quest to understand how students learn online"[14].

Based on Gartner's predictions there is likely to be, not only significant changes in adaptive learning methods, but also valuable sources of data mining activities to develop metrics and predictors of student behaviours and preferences when engaging in online learning. This assertion is further supported by Professor Jane Den Hollander, Vice Chancellor at Deakin University in Australia who suggests “... it will positively disrupt traditional thinking within universities by encouraging them to focus on how they can provide education in innovative ways" [15]. The Kodak company, whose early innovations in developing the first film-based cameras, were also innovators of the early digital cameras that are ubiquitous today and is an example of a high-profile casualty of lagging behind, and ultimately succumbing to, disruptive technologies [16].

The notion of "disruptive technology" warrants closer examination as this is a recurrent theme in characterising failed businesses, and presents a significant challenge to educational institutions wishing to accommodate the advancement of MOOCs. What is evident at the outset is that all educational stakeholders should be prepared to, at least, shift their thinking from previously held views and understandings of the modern learning environment to incorporate new educational technologies that serve identified needs. One of the earlier identifiers of "disruptive technology", Clayton Christensen, coined the phrase in 1997 as it was associated with a technology enabler [17]. Christensen cautioned corporations that staying close to customers whilst focusing on how to "...serve your core business while finding new markets and watching out for new entrants in your blind spot" will support survival. This proposal, when applied to the LMS, highlights the need to maintain the core functionality of the LMS through continual development and upgrades, whilst at the same time being cognisant of new and enhanced educational technologies which may challenge or act as complimentary components of existing LMS structure, and accommodate them where possible.

A synthesis of Christensen's theory and its applicability to respond appropriately to required change is summed up succinctly by Lucas Jr and Goh [16] who assert that "the most important observation is that management has to recognize the threats and opportunities of new information and communications technologies and marshal capabilities for change". Offering further clarity on this topic they suggest that "this change effort involves attacking core rigidities and the culture of the organization, and bringing all levels of employees on board, or the change effort will fail". The level of suggested change within organisations, particularly those in the educational environment with strong traditional, cultural leanings are clearly within the scope of Christensen's theory and therefore change, at least at some level, will be necessary for both educators and students.

One of the notable changes introduced by MOOC driven courses was in the way that new collaborative cultures emerged, which included Facebook, translations and discussions in a system that has been described as "a tool for democratizing higher education"[13]. Lewin notes that the automated nature of MOOCs currently deliver "... computer-graded assignment and exams" as well as incorporating question and answer sessions, voted on importance to draw attention to the lecturer and fostering a culture of sharing and social networking. These changes are partly accommodated by current LMS models but there is a need to look beyond the standard framework to provide a more enriching student experience.

As with the evolution of traditional websites, the initial LMS design architecture and content endures frequent changes. New iterations of the site morph the content through a patchwork of modifications in unexpected directions. The resulting product is often one far-removed from what was initially conceptualized at the outset. In some instances the LMS takes on the role of a portal as a convergence of related services (such as social networking), using the LMS as a gateway to access the additional tools. Such an approach highlights the somewhat slow evolution capabilities of the LMS.

Another key driver of new directions in educational technologies is the advancement of mobile technologies to enable collaborative activities among students [18]. The ubiquitous nature of mobile devices such as smart phones and tablets, and the "always connected" philosophy of students generates an expectation of a more direct and active relationship among all students and their lecturer. One of the notable benefits of deploying mobile technologies is that students can take advantage of the synchronous characteristics available to more closely interact with others through direct feedback quizzes, polls and surveys and provide a more engaging and collaborative learning experience.

A number of new mobile-device driven lecture feedback services have come onto the market in recent times. Examples include Poll Everywhere [19] and Top Hat Monocle [20] and these offer real-time capabilities including the ability to gauge student comprehension during lectures. In addition, the latter offers features such as the grading of student activities, thus providing a competing service with 
that offered by the LMS. A clear policy will need to be formulated within the education institution, to prevent a fragmentation of the educational services.

\section{NEW DiRECTIONS In LEARning MANAGEMENT SySTEMS}

Summarising the findings of a study to gauge user perceptions of the use of an LMS, Pace [21] states that for the most part, many people believe that it still fulfils its original function, that is, to track training, but, they are no longer using it just for that purpose. One of the most requested features of the LMS was "personalised learning plans" thus suggesting a more contributory, targeted and self-directed focus is needed. This requirement could be served through the use of specific technology systems which provide a useful vehicle to deliver those elements. The traditional LMS, as a stand-alone service, does not typically include the full capability to offer a complete range of services, thus requiring "add-on" features to fulfil specifications.

In response to the perceived limitations of the current LMS environment, Pace [21] suggests two possible key outcomes exist and should be incorporated into the future strategy of the LMS. The first option is to simply accept the existing capabilities (and limitations) of the LMS and continue to use it as it was designed to be used. The second option is to "integrate the LMS with new systems to get the job done". The former option clearly does not provide any evolutionary scope to meet the needs of the students and staff who use the LMS and thus does not appear to be a viable solution. The latter provides a more practical solution due to its flexibility and capacity to more closely align itself with the evolutionary requirements of the LMS and the software development lifecycle. The management, selection and integration of new and emerging systems will however need to be carefully monitored to ensure that only appropriate and compatible sub-systems are introduced into the LMS to ensure that no software conflicts occur or compatibility issues arise. As new complimentary software systems become available, there is likely to be increasing complexity in integrating these services within the LMS, so additional resources and skills may be needed to address any issues that arise.

With the increasing availability of complex, complimentary services such as synchronous feedback lecture tools to provide instant, in-class responses, educators are currently faced with several challenges in identifying and accommodating new LMS enhancements. The first challenge relates to keeping abreast of new developments in synchronous learning tools, particularly those extraneous to the LMS, and secondly, to master the use of those tools that evolve naturally as enhancements and new releases to the LMS which they are using. An examination of each of these challenges is needed to gain a deeper understanding of their composition and impact on the learning process and its stakeholders.

In relation to stand-alone, synchronous tools, academic staff may choose to conduct research into new and emerging technologies, and where they may be of use, introduced frequently through pilot implementations to evaluate their overall value to the teaching process. This process is, however, likely to be time consuming and beyond the scope of the staff member's teaching duties. The academic staff member's need to conduct independent research may be attributable to a slowness on the part of the LMS custodians to react to or even identify change as it occurs within the broader educational technology environment.

Where the tools are "pushed" to the academic staff member from within the LMS during a coordinated, production rollout within an academic institution, these are often accompanied by formal training and supporting reference literature to offer a more seamless learning experience for those involved. In many instances, internal stakeholders of the LMS within the organisation will have identified issues that may improve the overall functionality of the LMS, however, not immediately implement them. In view of the rigorous nature of information services-based changes and upgrades, factoring in critical elements of the development, testing and final release, the process of implementing new systems will likely be slow and thus perceived as being unresponsive to the needs of the users.

Both of the models of research and internally directed projects to introduce LMS enhancements are not dichotomous and could thus be pursued concurrently. On its own, the option of independent research may not benefit from the rigour of a more structured and active evolution of an existing LMS development programme. In many instances, structured, strategically-driven upgrade programmes are aligned with broader, technical information architecture initiatives, thereby benefitting from a clear roadmap to address future requirements that fit within the corporate network structure.

Based on the potential benefits derived from both independent research and a structured, strategically-driven LMS enhancement programme, a potential solution could incorporate both elements to take advantage of the respective value of each. A likely scenario for the advancement of a sustainable model to accommodate the practical requirements of an upgraded LMS, and user requirements may thus lie on a continuum between continued research and the structured, ongoing upgrading of the core LMS framework.

\section{CONCLUSION}

With the underpinning objective being to provide an enhanced learning outcome to students and academic staff members who use the LMS as a vehicle for learning, social and academic engagement, collaboration and research, it is important to tailor any future direction of the LMS to accommodate these elements as part of the overarching strategic direction. To this end, a firm roadmap should be formulated that engages communities of practice within the software development and user group network. This approach would allow the pooling of a collective need for emerging systems and services that have a place within the LMS environment, and which could be developed through the collective ideas and input from a development state into a live production environment.

As a primary source for useful requirements identification and feedback on the current state and student perceptions of the LMS as a learning tool would be the academic staff who use and receive commentary and feedback from students on 
an ongoing basis. The information channelled in this way would provide a platform to shape the information received and feed it, in turn, to other members of the LMS user and stakeholder community. Whilst the drive should be towards an enhanced system, realistic goals and expectations should be set with time-lines and milestones commensurate with the ability of the organisation to change and adapt to new technologies as “today's LMS is not nimble enough to meet all users' knowledge demands" [9].

In many ways, the development of the LMS has evolved slowly but yet remained functional and unquestionably served its intended purpose since its inception at the early, formative days of distance learning. The time has come, however, for a review of both the successful and unsuccessful services it provides in the contemporary learning space. The "disruptive technologies" are gathering momentum and if the LMS does not evolve efficiently and swiftly to meet the needs of its users, it may be replaced by a more efficient platform and a system that responds more closely to user needs.

With the current practice of incorporating complementary services within the LMS to improve the learning experience now coming into question in terms of its sustainability, Pace [21] succinctly asks the question: "What technology will overpower the LMS, or will the LMS add enough new technology to overcome these challenges?".

\section{REFERENCES}

[1] C. Christensen, "Disruptive innovation," Leadership Excellence, vol. 24, pp. 7, 2007.

[2] T. Clancy, G. Johnson, and T. Kelly. (May 18, 2013). What's the Future of the [Online]. Available: http://www.expertus.com/node/448.

[3] J. D. Hollander. (January 26, 2013). MOOCs: Neither the death of the university nor a panacea for learning. [Online]. Available: http://theconversation.edu.au/moocs-neither-the-death-of-the-universit y-nor-a-panacea-for-learning-8499.

[4] F. Ding, "eLearning and social media in higher education with an interactional approach," Academic Conferences International Limited, Kidmore End, United Kingdom, 126-XII, Oct. 2012.

[5] S. Downes. (May 17, 2013). The Buntine Oration: Learning Networks. [Online]. Available: http://www.downes.ca/cgi-bin/page.cgi?post=20

[6] S. Downes. (2005). E-Learn 2.0. eLearn Magazine. [Online]. Available: http://elearnmag.acm.org/featured.cfm?aid=1104968

[7] D. Gillmo. (May 18, 2013). We the Media. [Online]. Available: http://www.authorama.com/we-the-media-3.html

[8] L. S. Green, F. A. Inan, and B. Denton, "Examination of factors impacting student satisfaction with a new learning management system," Turkish Online Journal of Distance Education (TOJDE), vol. 13, pp. 189-197, 2012.
[9] N. Karousos, N. Kostaras, E. Koulocheri, A. Soumplis, and M. Xenos, "Learning management systems and learning 2.0," International Journal of Web-Based Learning and Teaching Technologies, vol. 6, no. $1,2011$.

[10] T. Lewin. (May 18, 2013). Instruction for Masses Knocks Down Campus Walls. New York Times. [Online]. Available: http://www.nytimes.com/2012/03/05/education/moocs-large-coursesopen-to-all-topple-campus-walls.html?pagewanted=all\&_r=0

[11] H. C. Lucas Jr and J. M. Goh, "Disruptive technology: How Kodak missed the digital photography revolution," The Journal of Strategic Information Systems, vol. 18, pp. 46-55, 2009.

[12] F. Mahnegar, "Learning management system," International Journal of Business and Social Science, vol. 3, no. a. 2012.

[13] M. Nehme, E-learning and Student's Motivation, 2010.

[14] A. Pace, What's Next for the LMS? T+D, vol. 65, pp.16, 2011.

[15] L. Pappano. (January 26, 2013). The Year of the MOOC. [Online]. Available:

http://www.nytimes.com/2012/11/04/education/edlife/massive-open-o nline-courses-are-multiplying-at-a-rapid-pace.html?pagewanted=all\& r $=0$

[16] Polleverywhere. (2013). How Poll Everywhere Works. [Online] Available: http://www.polleverywhere.com/how-it-works

[17] B. Rust, A. Weiner, M. Harris, and J. M. Lowendahl. (January 26, 2013). Predicts 2013: Digitalization Powers Education. [Online]. Available:

http://my.gartner.com/portal/server.pt?open=512\&objID=260\&mode $=2 \&$ PageID=3460702\&resId=2247715\&ref=QuickSearch \&sthkw=m ooc

[18] D. Taylor. (May 19, 2013). Does the LMS have a future? [Online]. Available:

http://donaldhtaylor.wordpress.com/2013/03/18/does-the-lms-have-a-f uture/

[19] Tophatmonocle. (May 21, 2012). Classroom Engagement Features. [Online]. Available: https://tophat.com/features

[20] P. Venter, M. J. V. Rensburg, and A. Davis, "Drivers of learning management system use in a South African open and distance learning institution," Australasian Journal of Educational Technology, vol. 28, pp. 183-198, 2012.

[21] D. Zhang, J. L. Zhao, L. Zhou, and J. F. Nunamaker, "Can e-learning replace classroom learning?" Communication. ACM, vol. 47, pp. 75-79, 2004.

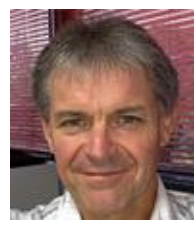

Ross Yates initially tertiary trained in human resources and spent several years as an HR practitioner in both South Africa and Australia. Dr Ross Yates has been involved in multi-faceted academic activities, underpinned by hands-on experience in IT business processes and project management methodologies acquired in a contemporary corporate environment. In addition to the academic roles, Dr Yates' immediate past role was a learning systems and training practitioner in the resources sector. Academic credentials include holding a postgraduate lecturing role at Edith Cowan University as well as a teaching and research role at Curtin University, Specialising in online privacy, virtual communities, e-Learning, website accessibility and usability Dr Yates' doctoral dissertation explored the role of privacy in cyberspace and the impact of privacy erosion through transactional surrender and gain. He has published refereed journal and conference papers supporting his work. 